\title{
Rapid Eye Movement Sleep and Hippocampal Theta Oscillations Precede Seizure Onset in the Tetanus Toxin Model of Temporal Lobe Epilepsy
}

\author{
Madineh Sedigh-Sarvestani, ${ }^{1}$ Godfrey I. Thuku, ${ }^{1}$ Sridhar Sunderam, ${ }^{1}$ Anjum Parkar, ${ }^{1}$ Steven L. Weinstein, ${ }^{5}$ \\ Steven J. Schiff,, ${ }^{1,2,3}$ and Bruce J. Gluckman ${ }^{1,2,4}$ \\ Center for Neural Engineering, Departments of ${ }^{1}$ Engineering Science and Mechanics, ${ }^{2}$ Neurosurgery, ${ }^{3}$ Physics, and ${ }^{4}$ Bioengineering, Pennsylvania State \\ University, University Park, Pennsylvania 16802, and ${ }^{5}$ Children's National Medical Center, George Washington University, Washington, DC 20010
}

\begin{abstract}
Improved understanding of the interaction between state of vigilance (SOV) and seizure onset has therapeutic potential. Six rats received injections of tetanus toxin (TeTX) in the ventral hippocampus that resulted in chronic spontaneous seizures. The distribution of SOV before 486 seizures was analyzed for a total of $19 \mathrm{~d}$ of recording. Rapid eye movement sleep (REM) and exploratory wake, both of which express prominent hippocampal theta rhythm, preceded 47 and $34 \%$, for a total of $81 \%$, of all seizures. Nonrapid eye movement sleep (NREM) and nonexploratory wake, neither of which expresses prominent theta, preceded 6.8 and $13 \%$ of seizures. We demonstrate that identification of SOV yields significant differentiation of seizure susceptibilities, with the instantaneous seizure rate during REM nearly 10 times higher than baseline and the rate for NREM less than half of baseline. Survival analysis indicated a shorter duration of preseizure REM bouts, with a maximum transition to seizure at $\sim 90 \mathrm{~s}$ after the onset of REM. This study provides the first analysis of a correlation between SOV and seizure onset in the TeTX model of temporal lobe epilepsy, as well as the first demonstration that hippocampal theta rhythms associated with natural behavioral states can serve a seizure-promoting role. Our findings are in contrast with previous studies suggesting that the correlations between SOV and seizures are primarily governed by circadian oscillations and the notion that hippocampal theta rhythms inhibit seizures. The documentation of significant SOV-dependent seizure susceptibilities indicates the potential utility of SOV and its time course in seizure prediction and control.
\end{abstract}

Key words: epilepsy; hippocampal theta; preseizure state; rapid eye movement; sleep; tetanus toxin

\section{Introduction}

There is an acknowledged relationship between state of vigilance (SOV), also known as sleep-wake state, and seizure onset in various human (Bazil and Walczak, 1997; Crespel et al., 2000; for review, see Dinner, 2002; Minecan et al., 2002; Malow, 2007; Kothare and Zarowki, 2011; Sinha, 2011) and experimental (Quigg et al., 2000; Shouse et al., 2000; Colom et al., 2006; for review, see Matos et al., 2011; Sinha, 2011) epilepsies. Additionally, epilepsy, in turn, alters sleep patterns (Cepeda et al., 1982; Ayala-Guerro et al., 2002; Matos et al., 2010; for review, see Matos et al., 2011).

Different SOVs are accompanied by changes in synaptic transmission and patterns of network activity in the brain (Lodden-

\footnotetext{
Received July 19, 2013; revised Dec. 4, 2013; accepted Dec. 6, 2013.

Author contributions: M.S.-S., S.S., S.L.W., S.J.S., and B.J.G. designed research; M.S.-S., G.I.T., and A.P. performed research; M.S.-S., S.L.W., S.J.S., and B.J.G. analyzed data; M.S.-S., S.J.S., and B.J.G. wrote the paper.

This work was supported by National Institutes of Health Grants 1F31NS070545 (M.S.-S.), R03NS065451 (S.S.), and R01NS065096 (B.J.G., S.J.S.).

The authors declare no competing financial interests.

Correspondence should be addressed to Madineh Sedigh-Sarvestani, Department of Neuroscience, University of Pennsylvania, Philadelphia, PA 19104. E-mail: msarvestani@gmail.com.

G. I. Thuku's present address: Department of Surgery, Virginia Commonwealth University, Richmond, VA. S. Sunderam's present address: Center for Biomedical Engineering, University of Kentucky, Lexington, KY. DOI:10.1523/JNEUROSCI.3103-13.2014

Copyright $\odot 2014$ the authors $\quad 0270-6474 / 14 / 341105-10 \$ 15.00 / 0$
}

kemper et al., 2011; Sinha, 2011). These widespread changes likely affect other network dynamics, such as seizures. Thus, although we do not yet understand the mechanisms of this interaction, the observed effect of SOV on seizure onset is not surprising given our knowledge of sleep-wake networks. Despite the availability of several animal models of epilepsy (Buckmaster, 2004; Soltesz and Staley, 2008), the effect of sleep in epilepsy remains relatively unexplored (Malow, 2007; Matos et al., 2011; Dewolfe et al., 2013).

Improved insight into the interaction between SOV and seizure onset may help to identify novel pharmaceutical therapies and enhance stimulation-based seizure intervention strategies (Loddenkemper et al., 2011; Dewolfe et al., 2013). There is consensus that epileptic seizures often arise from nonrapid eye movement sleep (NREM) and relaxed wake and rarely from rapid eye movement sleep (REM) or active wake states in animals (Miller et al., 1994; Shouse et al., 2000; Colom et al., 2006) and humans (Crespel et al., 2000; Herman et al., 2001; Minecan et al., 2002; Malow 2007; Ng and Pavlova, 2013). However, the relationships between SOV and seizure onset seem to be dependent on the etiology of the epilepsy model or syndrome.

The aim of this study was to quantify the relationship between SOV and seizure onset in the rat tetanus toxin (TeTX) model of temporal lobe epilepsy (TLE). To date, there have been no pub- 

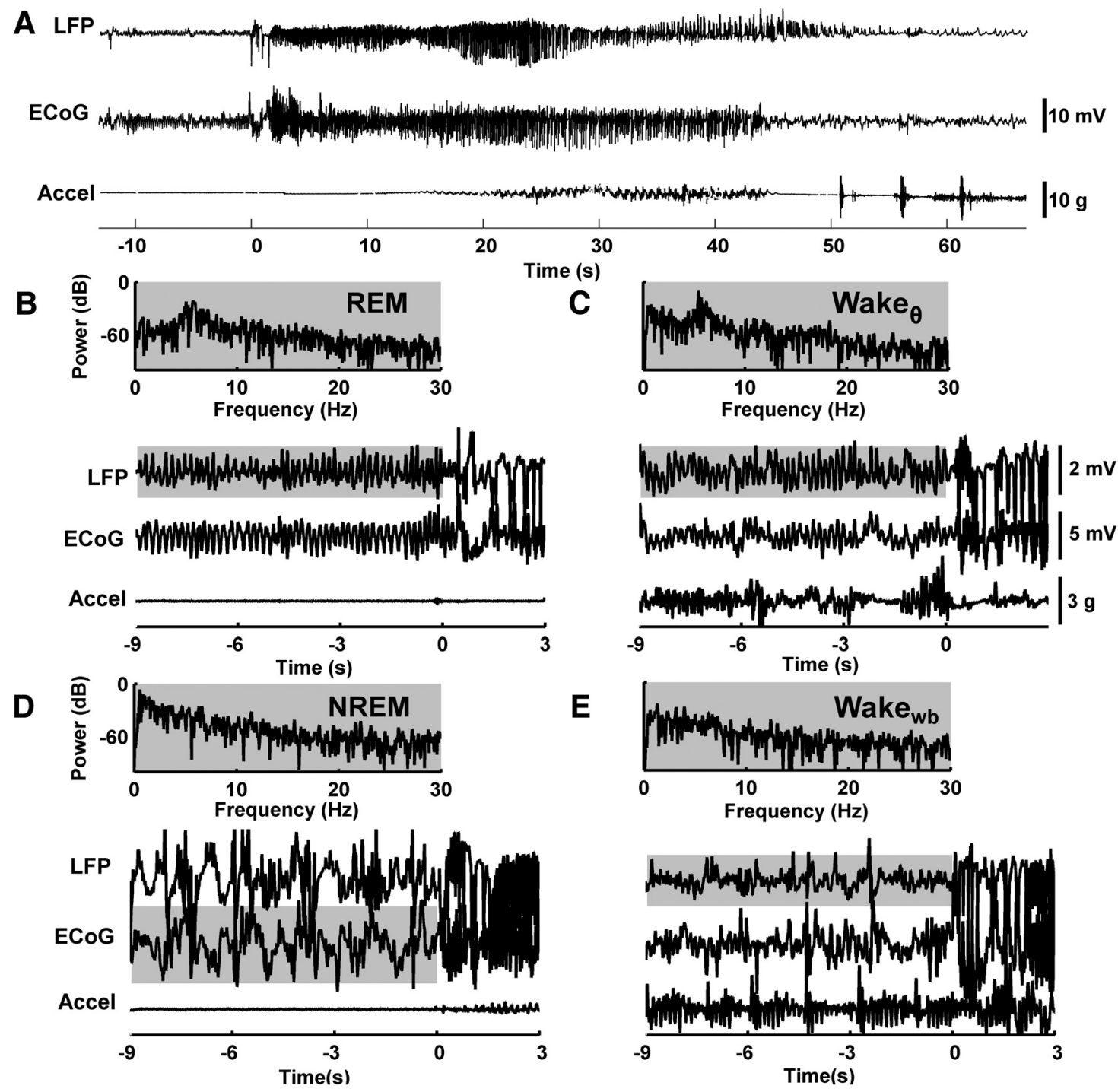

Figure 1. Typical seizure and SOV traces. $A$, Typical seizure. Time-series traces of LFP recorded from the left dorsal hippocampus, ECOG recorded from the surface of the right hemisphere, and head acceleration (Accel). For display purposes, LFP and ECOG traces were bandpass filtered between 0.5 and $35 \mathrm{~Hz}$. The seizure starts at 0 s. This seizure ranked as a stage 5 seizure on the Racine scale. $\boldsymbol{B}-\boldsymbol{E}$, Time and power series for different SOVs, with seizures initiating at $0 \mathrm{~s}$. The power spectrum is shown for the highlighted portion of time-series trace. $\boldsymbol{B}$, REM is defined by rhythmic hippocampal activity in the theta band $(4-12 \mathrm{~Hz})$, lack of activity in the delta band $(0.5-4 \mathrm{~Hz})$, and with muscle atonia interrupted with brief muscle twitches, which are reflected in measures of head acceleration. C, Wake ${ }_{\theta}$ is defined by rhythmic hippocampal activity in the theta band along with high Accel activity indicating locomotion. D, NREM is defined by cortical activity in the delta band, lack of activity in the theta band, and lack of motion indicative of sleep. $\boldsymbol{E}$, Wake ${ }_{\mathrm{wb}}$ is characterized by either low- or high-power Accel activity indicating lack or presence of head movement, respectively, and broadband hippocampal spectrum in the range of $0.5-30 \mathrm{~Hz}$.

lished studies on the effect of SOV on seizure onset in this model. TeTX cleaves synaptobrevin (Schiavo et al., 1992), a protein necessary for neurotransmitter release. When injected into the rodent brain, TeTX reduces neurotransmitter release, primarily from inhibitory neurons (Britton et al., 1996; Mainardi et al., 2012). This leads to short-term (Empson et al., 1993) and longterm (Vreugdenhil et al., 2002) changes in excitability, which manifest as chronic epilepsy, with little hippocampal cell loss or mossy fiber sprouting (Jefferys and Walker, 2006; Sharma et al., 2007).

Our primary finding is that, in the TeTX model of TLE, a disproportionately large fraction of seizures occurred during REM and active wake periods exhibiting hippocampal theta oscillations and that a disproportionately small fraction of seizures occurred during NREM and relaxed wake periods. These findings support the notion that in addition to NREM-associated cortical slow oscillations, REM- and active wake-associated hippocampal theta oscillations can serve a seizure-promoting role.

\section{Materials and Methods}

All work was approved and performed under the oversight of the Pennsylvania State University Institutional Animal Care and Use Committee.

Animal surgery and care. Male Long-Evans rats, weighing 275-350 g, were implanted with recording electrodes and given injections of TeTX during the same surgical procedure. Two pairs of stainless steel screws were used for the differential measurement of the electrocorticogram (ECoG) at coordinates [anteroposterior (AP), ML] of $[0.5, \pm 3]$ and $[-7$, \pm 3 . For the differential measurement of local field potential (LFP), four pairs of $50 \mu \mathrm{m}$ iridium oxide-coated stainless steel wire electrodes with ends staggered by $300 \mu \mathrm{m}$ were implanted bilaterally in the dorsal hippocampus at coordinates $(\mathrm{AP}, \mathrm{ML})[-2.5, \pm 2]$ and $[-3.9, \pm 2.2]$. Electrodes were positioned, close to $-2.8 \mathrm{DV}$, such that population firing activity of CA1 neurons was evident. All coordinates are in millimeters, 
A
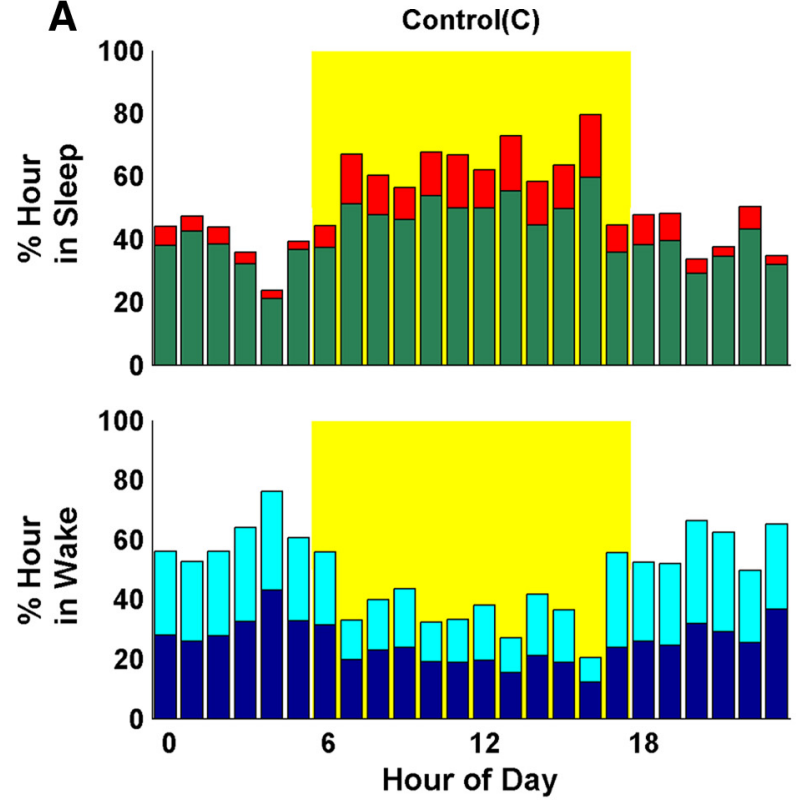

B
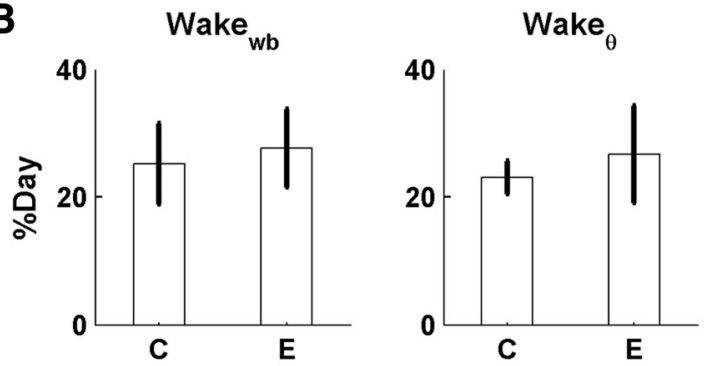
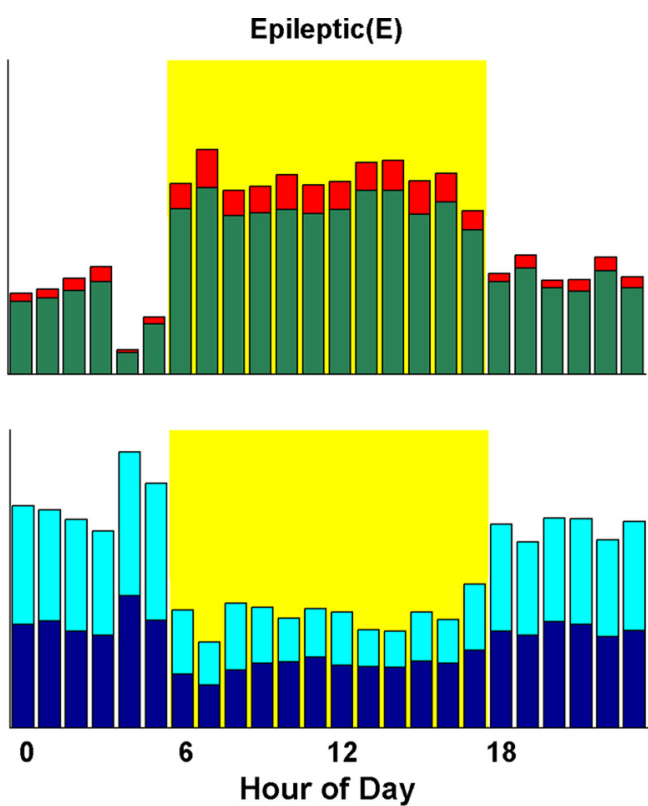

NREM

REM
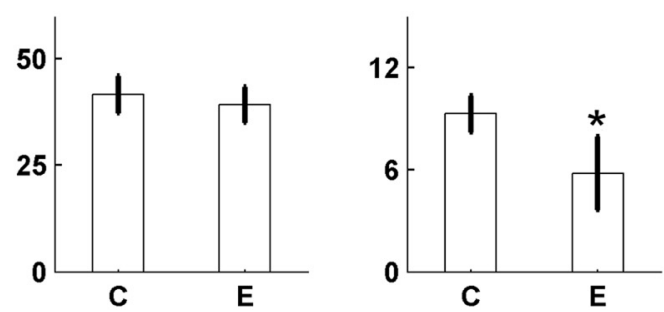

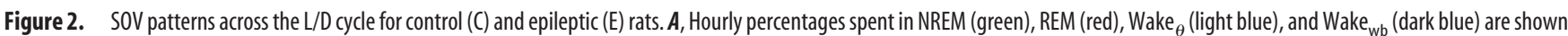
for control and epileptic rats. B, Mean and SD of percentage of day spent in each SOV. The difference in group distributions was calculated using a two-sided unequal variance $t$ test with $\alpha=0.05$. Epileptic rats exhibit a significant decrease in daily REM time compared with nonepileptic controls.

and bregma is referenced according to Paxinos and Watson (2006). Ten nanograms of TeTX (Sigma-Aldrich), dissolved in $1 \mu \mathrm{l}$ of a $2 \%$ bovine serum albumin/0.05 м PBS (Sigma-Aldrich) solution, were injected in the ventral hippocampus at coordinates (AP, ML, DV) of $[-5.15,5.35$, $-7.65]$. Electrodes were secured in place with cyanoacrylate and electrically isolated with dental cement.

Rats were housed individually in custom-made Plexiglas cages with dimensions 9 (width) $\times 15$ (depth) $\times 24$ (height) inches. A counterbalance system, including a commutator, attached to the head-mounted electronics allowed for postural flexibility and free movement. Rats were allowed to recover for a period of 3 to $7 \mathrm{~d}$ after surgery and had ad libitum access to food and water. A $12 \mathrm{~h}$ light/dark (L/D) cycle was maintained with the lights on between 6:00 A.M. and 6:00 P.M. During the dark period, infrared LED arrays were used to provide illumination for continuous video monitoring.

Data collection. Video monitoring, at three frames per second, began on the day of implantation. After the recovery period, implanted rats were cabled to recording electronics, including a head-mounted preamplifier with tri-axial accelerometers (Sunderam et al., 2007) used to monitor head motion and orientation. All signals were amplified by $20-$ at the head-mounted preamplifier and filtered at $0.3-800 \mathrm{~Hz}$ at a second recording amplifier mounted exterior to the animal's cage. Signals were sampled with 16-bit resolution at $2 \mathrm{kHz}$ using an acquisition board (National Instruments).

Data analysis. At least $24 \mathrm{~h}$ of data were analyzed for each rat. Longer data-sets, up to $7 \mathrm{~d}$, were used when available. For some rats, stimulation experiments followed the observation period. No poststimulation data were included in this study to avoid potential confounding effects. Inclusion criteria for each hour of data were as follows: availability of clear video recording, at least one low-noise depth LFP recording, at least one low-noise ECoG recording, and at least one low-noise recording of head acceleration. We also required either a LFP or ECoG recording from both hemispheres to monitor bilateral sleep and generalized seizure activity.

Seizures were detected automatically using in-house software and verified visually to determine more precise onset and end times. Seizure onset was defined, with a resolution of $1 \mathrm{~s}$, as the first instance where the power of the LFP recordings was at least three times higher than the average background power. Unilateral seizures that failed to generalize $(n=2)$, seizure events that were $<10 \mathrm{~s}$ in duration $(n=11)$, and seizure events that occurred within $5 \mathrm{~min}$ after the onset of the previous seizure $(n=27)$ were excluded from analysis. LFP and ECoG traces, as well as an accelerometer trace, are shown for a typical seizure in Figure $1 A$.

SOV was scored in nonoverlapping $10 \mathrm{~s}$ windows according to the algorithm described by Sunderam et al. (2007) using at least $4 \mathrm{~h}$ of visually scored training data for each $24 \mathrm{~h}$ to be scored for each rat. SOV was marked as one of the four states shown, before the onset of seizure at $0 \mathrm{~s}$, in Figure 1: REM (Fig. 1B), NREM (Fig. 1D), Wake with high activity in the hippocampal theta $(4-12 \mathrm{~Hz})$ band $\left(\mathrm{Wake}_{\theta}\right)$ (Fig. 1C), and Wake with wide-band hippocampal spectrum $\left(\mathrm{Wake}_{\mathrm{wb}}\right.$ ) (Fig. 1E). REM was characterized by a spectral peak in the theta band of hippocampal LFP and by an absence of accelerometer activity except during brief muscle twitches. NREM was characterized by maximal power in the ECoG delta $(0.5-4 \mathrm{~Hz})$ band and by an absence of accelerometer activity. Wake ${ }_{\theta}$ was characterized by a spectral peak in the theta band of hippocampal LFP and high accelerometer activity. Wake ${ }_{w b}$ was characterized by either lowor high-power accelerometer activity and by lack of LFP spectral peaks in the range of $0.5-30 \mathrm{~Hz}$. 
Performance on out-of-sample data was visually verified for each animal, and more training data were added until automated accuracy exceeded $85 \%$. In general, the algorithm classified SOV correctly with the exception of labeling some $W_{a k e}$ wb periods as NREM and the beginning of some NREM periods as Wake ${ }_{\mathrm{wb}}$. REM, Wake ${ }_{\theta}$, and post-transition NREM were rarely misclassified.

We define a "bout" as a continuous period in a single SOV, with no transitions to another SOV that lasts longer than $3 \mathrm{~s}$. Bout durations were calculated using automated scores and verified visually to achieve improved temporal resolution finer than $10 \mathrm{~s}$. Onset and end of REM was defined, with a resolution of $1 \mathrm{~s}$, within the first and last consecutive $10 \mathrm{~s}$ window during which a clear peak in the theta band was distinguishable and no head motion was seen on the accelerometers or the video.

Preseizure SOV was rescored for the $300 \mathrm{~s}$ before seizure in $10 \mathrm{~s}$ windows aligned with seizure onset. Multitaper spectral analysis, implemented in Matlab using the Chronux 2.0 toolbox (Bokil et al., 2010), was used to obtain estimates of theta and delta band powers needed to compute $\theta / \delta$ ratios. The $\theta / \delta$ ratio was computed by dividing the total power in the theta band by the total power in the delta band in nonoverlapping $10 \mathrm{~s}$ windows.

We used survival analysis with KaplanMeier estimates to calculate the hazard rate of transitioning out of a REM bout at time $t$, given that the REM bout has lasted up to time $t$. We calculated hazard rates for REM bouts separately from control and epileptic animals. We further grouped REM bouts from epileptic animals into those that ended in seizure (preseizure REM) and those that ended in Wake or NREM (interseizure REM). Because a transition to seizure precludes a transition to Wake or NREM, we used a competing risk framework (Furstova and Valenta, 2011). Bootstrap sampling was used to assess statistical significance of observed differences between the preseizure and interseizure hazard rates.

\section{Results}

Data from six rats were pooled to study the temporal correlation between SOV and seizure onset. Each rat had at least $24 \mathrm{~h}$ of analyzed data and at least 10 seizures per day, with a total of $19 \mathrm{~d}$ of data and 486 seizures. To determine whether seizures had a significant effect on sleep-related dynamics, we scored 72 consecutive hours for each of two weight-matched control rats. These rats underwent an electrode implant protocol identical to that for epileptic rats but did not receive TeTX injections.

\section{Effect of seizures on SOV}

Hourly percentages spent in each of four SOVs are shown for control and epileptic rats in Figure 2. Red denotes REM, green denotes NREM, light blue denotes Wake ${ }_{\theta}$, and dark blue denotes Wake $_{\mathrm{wb}}$. This color scheme is used for all subsequent figures. Epileptic and nonepileptic controls rats exhibited similar diurnal variation in activity and SOV as expected for nocturnal animals. The daily fraction of time spent in NREM and Wake was not overtly different between the control and epileptic groups. However, the daily fraction of time spent in REM was lower for epi- leptic rats compared with controls $[5.8 \pm 2.1 \%(\mathrm{SD})$ and $9.3 \pm$ $1.1 \%$ (SD); $p<0.01$, two-tailed $t$ test $]$, where averages are across days. This deviation coincides with epileptic rats exhibiting fewer REM bouts than control rats.

\section{Effect of L/D cycle and SOV on seizure onset}

Rats began to seize 3-10 d after TeTX injection. To allow sufficient recovery time for animals, data were recorded between 8 and $16 \mathrm{~d}$ after implant. Seizures typically had motor manifestations including rearing and loss of posture corresponding to a score of 4 or 5 on the Racine scale (Racine, 1972).

Seizures were not uniformly distributed throughout the day. As shown in Figure $3 A$, more seizures occurred during the light period compared with the dark period. The light-period seizure rate of $1.3 \pm 0.32$ seizures per hour $(\mathrm{sz} / \mathrm{h})(\mathrm{SD})$ was $63 \%$ higher than the dark-period rate of $0.84 \pm 0.34 \mathrm{sz} / \mathrm{h}(\mathrm{SD})$. To decouple this diurnal variation in seizure rate from the diurnal variation in SOV shown in Figure 2, we considered trends in preseizure SOV together with trends in the amount of time spent in each SOV across the L/D cycle. In Figure 3, we give these statistics for individual rats and for the group-weighted average, computed by averaging across the individual rat's statistic weighted by each rat's recording time. Unless otherwise noted, the group statistics are discussed. 
A

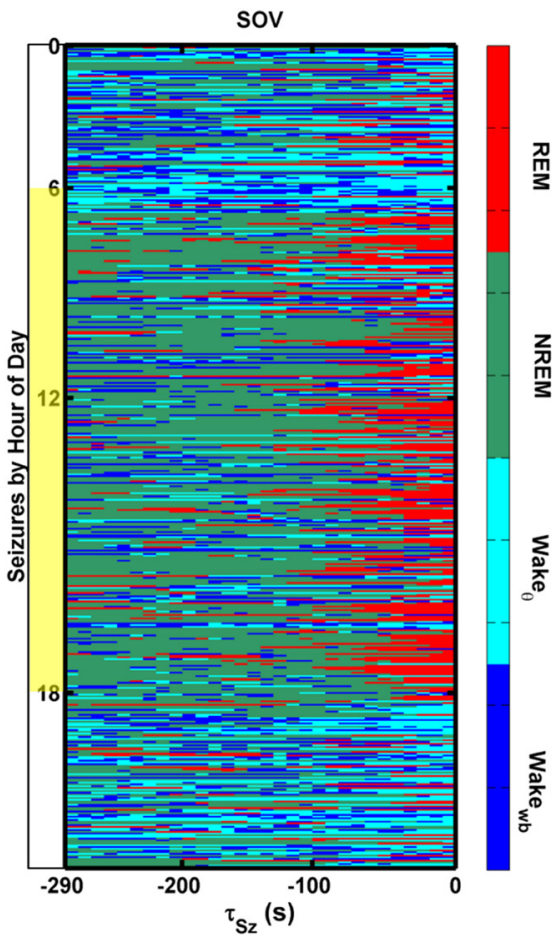

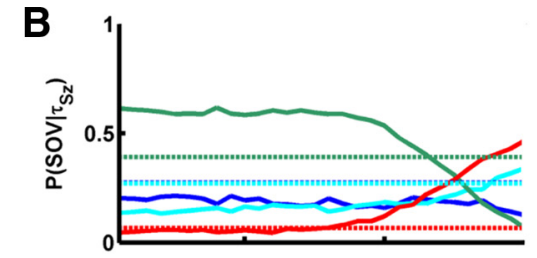

篮

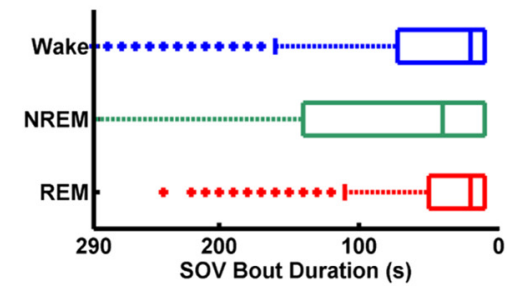

Figure 4. Preseizure SOV.A, SOV, scored in $10 \mathrm{~s}$ windows, is shown for the $300 \mathrm{~s}$ preceding each seizure as a function of time to seizure onset $\tau_{\mathrm{sz}}$. Seizures from all rats are sorted according to the hour of day during which they occurred. During the light period (in yellow), seizures are most often preceded by REM, which, in turn, is preceded by NREM. During the dark period (in white), seizures are most often preceded by Wake ${ }_{\theta}$, which is usually preceded by Wake ${ }_{\mathrm{wb}}$. $\boldsymbol{B}$, Probability of SOV as a function of $\boldsymbol{\tau}_{\mathrm{Sz}}$ $P\left(\mathrm{SOV} \mid \boldsymbol{\tau}_{\mathrm{S}_{2}}\right)($ top). Dashed lines indicate $P(\mathrm{SOV})$, or a fraction of all time spent in each SOV. Seizure rate conditioned on SOV, $R\left(\boldsymbol{\tau}_{\mathrm{S}_{\mathrm{Z}}} \mid \mathrm{SOV}\right)$ (bottom). The unconditioned seizure rate, $R\left(\tau_{\mathrm{S}_{\mathrm{Z}}}\right)$, is shown as black dashed lines. $R\left(\boldsymbol{\tau}_{\mathrm{Sz}} \mid \mathrm{SOV}\right)$ for $\mathrm{SOV}=\left\{\mathrm{Wake}_{\theta^{\prime}}\right.$ Wake $_{w b}$, NREM $\}$ is close to $P\left(\tau_{s_{z}}\right)$. However, $R\left(\tau_{S_{z}} \mid R E M\right)$ starts to rise at 130 s and reaches nearly 10 times the unconditioned seizure rate at seizure onset. Units for seizure rates are ${ }^{-1}$. C, Bout duration box and whisker plots for each SOV. Wake ${ }_{w b}$ and Wake ${ }_{\theta}$ were pooled as one state. The range of REM-bout duration is [10-120] seconds and overlaps with the increase in $R\left(\boldsymbol{\tau}_{\mathrm{Sz}} \mid R E M\right)$ just before seizure.

We found that $47 \%$ of seizures occurred during REM, with $38 \%$ in the light period and $9 \%$ in the dark period. The next greatest fraction of seizures (34\%) initiated in Wake ${ }_{\theta}$, with $12 \%$ during the light period and $22 \%$ during the dark period. Rats spend about three times as much time in REM during the light period as during the dark period. Thus, the increased fraction of seizures arising from REM during the light period is coincident with an increase in the fraction of time spent in REM, as illustrated in Figure 3B. Similarly, the increased fraction of seizures arising from Wake ${ }_{\theta}$ during the dark period is coincident with an increase in the fraction of time spent in this state.

Only $6.8 \%$ of all seizures occurred during NREM. This was unexpected because $39 \%$ of time was spent in NREM (L/D) and because prior findings attributed a seizure-promoting role to NREM.

\section{Preseizure SOV distribution}

To gain insight into the predictive utility of SOV, we considered the SOV distribution for a preseizure period of $5 \mathrm{~min}$. The colorcoded distribution of SOV as a function of time to seizure onset $\left(\tau_{\mathrm{Sz}}\right)$, for all seizures from all rats, is plotted in Figure 4 A. Seizures were sorted according to the hour of day in which they occurred. Each pixel represents the SOV for a $10 \mathrm{~s}$ window. During the light period, NREM bouts transition into REM bouts, which transition into seizures. During the dark period, seizures most often begin in $\mathrm{Wake}_{\theta}$, which is usually preceded by Wake ${ }_{\mathrm{wb}}$. In contrast to
REM bouts clustered close to seizure onset, Wake $\theta_{\theta}$ appears more uniformly throughout the preseizure window.

The fraction of seizures arising from each SOV as a function of $\tau_{\mathrm{Sz}}$ is shown as solid lines in Figure $4 B$ (top). We denote this fraction $P\left(\mathrm{SOV} \mid \tau_{\mathrm{Sz}}\right)$. Sufficiently ahead of seizures (i.e., for $\tau_{\mathrm{Sz}}>15 \mathrm{~min}$, data not shown $), P\left(\mathrm{SOV} \mid \tau_{\mathrm{Sz}}\right)$ converges to $P(\mathrm{SOV})$, the unconditioned probability of state shown as dashed lines. These probabilities are significantly different within the $5 \mathrm{~min}$ preseizure epoch shown, with $P(\mathrm{REM} \mid-10 \mathrm{~s})$ notably approaching onehalf as $P\left(\mathrm{NREM} \mid \tau_{\mathrm{Sz}}\right)$ approaches zero.

We can estimate the predictability of seizure (posterior probability) given the current SOV using the Bayes rule:

$$
P\left(\tau_{S z} \mid S O V\right)=\frac{P\left(S O V \mid \tau_{S z}\right) P\left(\tau_{S z}\right)}{P(S O V)},
$$

where $P\left(\tau_{\mathrm{Sz}} \mid \mathrm{SOV}\right)$ is the conditional probability of observing a seizure at future time $\tau_{\mathrm{Sz}}$ given that the current state is SOV. $P\left(\tau_{\mathrm{Sz}}\right)$ is the unconditioned probability of observing a seizure. Conditional seizure probabilities are reported as rates by multiplying by the SOV measurement rate of 360 per hour. This yields the predicted seizure rate, $R\left(\tau_{\mathrm{Sz}} \mid \mathrm{SOV}\right)$, future time $\tau_{\mathrm{Sz}}$ conditioned on the observed current SOV shown in Figure $4 B$ (bottom, solid lines). The unconditioned seizure rate, $R\left(\tau_{\mathrm{Sz}}\right)=P\left(\tau_{\mathrm{Sz}}\right) * 360$, is shown as the black dashed line.

For all states other than REM, $R\left(\tau_{\mathrm{Sz}} \mid \mathrm{SOV}\right)$ is close to the unconditioned seizure rate over the majority of the $5 \mathrm{~min}$ epoch before seizure. However, $R\left(\tau_{\mathrm{Sz}} \mid \mathrm{REM}\right)$ starts to rise $130 \mathrm{~s}$ ahead of seizure and reaches nearly 10 times the unconditioned rate just before seizure onset. We note, therefore, that observation of REM provides a short-term prediction: the probability that a seizure will occur within the next $130 \mathrm{~s}$, given that the current SOV is REM, is much higher than the unconditioned probability of seizure occurrence.

Bout duration distributions for each SOV are shown with box and whisker plots in Figure $4 C$. Wake ${ }_{\theta}$ is highly fragmented. Therefore, Wake ${ }_{\mathrm{wb}}$ and $\mathrm{Wake}_{\theta}$ were pooled to give just Wake bout durations. Box edges mark the first and third quartiles, or the interquartile range (IQR), and the middle line marks the median. Whiskers extend to the third quartile plus $1.5 *$ (IQR). Data points outside this range are considered outliers and are indicated by asterisks. The range of nonoutlier REM bout durations is $[10-120]$ seconds. This range correlates with the $130 \mathrm{~s}$ seizure-predictive window given observation of REM.

The seizure rate can be written as a linear model of the form $P\left(\tau_{S_{z}}\right)=\Sigma_{S O V} R\left(\tau_{S_{z}} \mid S O V\right) P(S O V)$. To determine whether the observed diurnal variation in seizure rate could be fully contributed to diurnal changes in SOV, we fit this model to the observed seizure time series and calculated $R\left(\tau_{\mathrm{Sz}} \mid \mathrm{SOV}\right)$. We found that constant SOV-conditioned seizure rates across the L/D cycle could not fully account for the observed $\mathrm{L} / \mathrm{D}$ variation in seizure rate, and so we calculated $R\left(\tau_{\mathrm{Sz}} \mid \mathrm{SOV}\right)$ separately for $\mathrm{L} / \mathrm{D}$. The 
weighted mean seizure rate during REM drops from $9.2 \pm 16 \mathrm{sz} / \mathrm{h}$ in the light period to $7.4 \pm 16 \mathrm{sz} / \mathrm{h}$ in the dark period, and in $\mathrm{Wake}_{\theta}$ is from $1.5 \pm 0.53 \mathrm{sz} / \mathrm{h}$ in the light to $1.2 \pm 0.26 \mathrm{sz} / \mathrm{h}$ in the dark period. In NREM, the $\mathrm{L} / \mathrm{D}$ rates are $0.18 \pm 0.028$ and $0.20 \pm 0.10$, and in $\mathrm{Wake}_{\mathrm{wb}}$, they are $0.78 \pm 0.25$ and $0.35 \pm 0.024 \mathrm{sz} / \mathrm{h}$. The high SDs for REM-related rates are attributable to the small and somewhat variable amount of time spent in this state. This variation of $R\left(\tau_{\mathrm{Sz}} \mid \mathrm{SOV}\right)$ across the L/D cycle indicates that the correlation between diurnal cycles and seizure frequency is independent of SOV. However, the L/Drelated variations are much smaller than the SOV-related variations in seizure frequency. As an example, the ratio between the mean light-period REM (9.2) and NREM (0.18) seizure rates is $51: 1$. In contrast, the ratio between the light-period (9.2) and dark-period (7.4) REM seizure rates is much smaller at 1.2:1. Thus, the inclusion of time of day in addition to the observation of current SOV will increase predictability, but only by a modest amount.

\section{Characteristics of preseizure REM and theta oscillations}

The spectral characteristics of hippocampal LFP before seizure onset, independent of SOV, is also instructive. As illustrated in Figure 5, the normalized power in thetaband LFP activity, as estimated from the $\theta / \delta$ ratio, increases, on average, $100 \mathrm{~s}$ ahead of seizure, in agreement with the observation that $80 \%$ of all seizures arise from either REM or $\mathrm{Wake}_{\theta}$. In Figure $5 A-C$, we present detailed analysis for only one rat (R6) to offer more resolution in the figures. In Figure $5 D$, we show the preseizure temporal trend of the $\theta / \delta$ for each rat. The mean and SD of $\theta / \delta$ ratio in the $300 \mathrm{~s}$ preseizure window for R6 are as follows: REM, 1.7 \pm 0.75; Wake $_{\theta}, 1.4 \pm 0.33$; NREM, $0.91 \pm 0.29$; Wake $_{\mathrm{wb}}, 0.76 \pm 0.17$. The average $\theta / \delta$ ratio $(1.2 \pm 0.58)$ over the entire data for R6, shown in the solid line in Figure $5 D$, is within the distribution of $\theta / \delta$ ratios for Wake ${ }_{\theta}$ in this animal. Because this animal spends $33 \%$ of its time in $\mathrm{Wake}_{\theta}$, the observation of high $\theta / \delta$ ratios alone does not provide significant predictability of seizure onset. Across all rats, we found that the SOV-conditioned $\theta / \delta$ ratio distribution did not vary with time to seizure onset (data not shown), suggesting that preseizure variations in the $\theta / \delta$ ratio are fully explained by preseizure SOV variation. The difference between the predictive utility of REM versus $\theta / \delta$ ratio is apparent from the comparison of the large deviation between $P$ (REM $\mid 32$ $\tau_{\mathrm{Sz}}$ ) and $P(\mathrm{REM})$ in Figure $5 C$ (red) with the relatively small deviation between the $\theta / \delta$ ratio in the $300 \mathrm{~s}$ preseizure window and the $\theta / \delta$ ratio over all time shown in Figure $5 D$.

Although nearly half of all seizures arise from REM, only $19 \%$ of all REM bouts lead to seizure. To gain insight into the mechanism that determines which REM bouts transition into seizure,
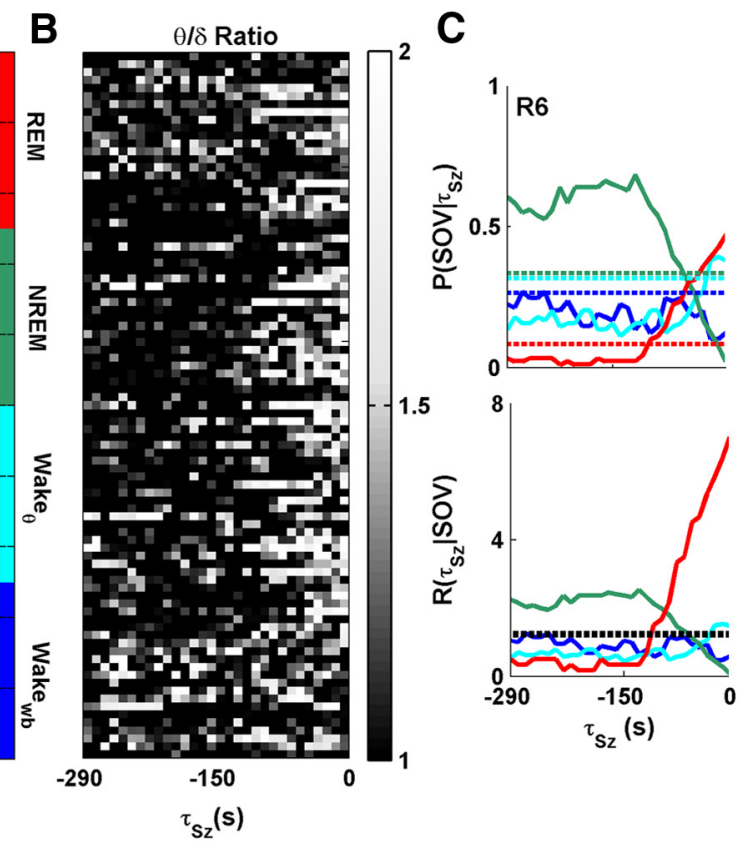

$\tau_{\mathrm{sz}}(\mathbf{s})$
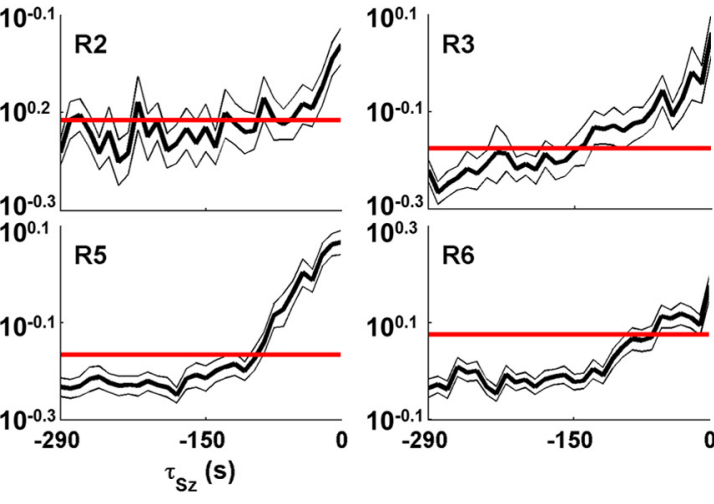

Figure 5. Preseizure SOV and hippocampal LFP $\theta / \delta$ ratios. $A$, SOV for 300 s before each seizure for rat 6 (R6). Seizures are sorted according to the hour during which they occurred. $\boldsymbol{B}$, The $\theta / \delta$ ratio for hippocampal LFP calculated for $10 \mathrm{~s}$ windows for same the average ratio for Wake ${ }_{\theta}$ is lower than that for REM epochs. NREM and Wake ${ }_{w b}$ epochs have low $\theta / \delta$ ratios. $C, P\left(\tau_{\mathrm{Sz}} \mid \mathrm{SOV}\right)$ for R6. surrounding the mean indicate SEM. Flat red lines indicate the average $\theta / \delta$ ratio over the entire recording period for each rat. For all rats, the $\theta / \delta$ ratio increases before seizure onset.

we compared spectral and temporal properties of the REMassociated theta oscillations across preseizure and interseizure REM bouts. Preseizure REM bouts $(n=224)$ always transitioned to seizure, and interseizure REM bouts $(n=970)$ always transitioned to the nonpathological states of Wake or NREM. We contrast these REM bout properties to those calculated from two nonepileptic control animals ( $n=569$ bouts).

Typical traces of REM from an epileptic and a control animal are shown in Figure 6A. Preseizure and interseizure REM bouts from the same rat are plotted separately in Figure $6 A$ (top and middle). The LFPs before the transitions out of REM in epileptic animals appear to be indistinguishable between seizure and Wake/NREM transition types. The characteristic theta oscillations of REM persist until seizure onset (Fig. 6A, top, vertical line), and no indication of a transition into another SOV is evident. The theta oscillations of REM in control animals, shown in Figure $6 \mathrm{~A}$ (bottom), do not appear overtly different than oscillations in epileptic rats. 


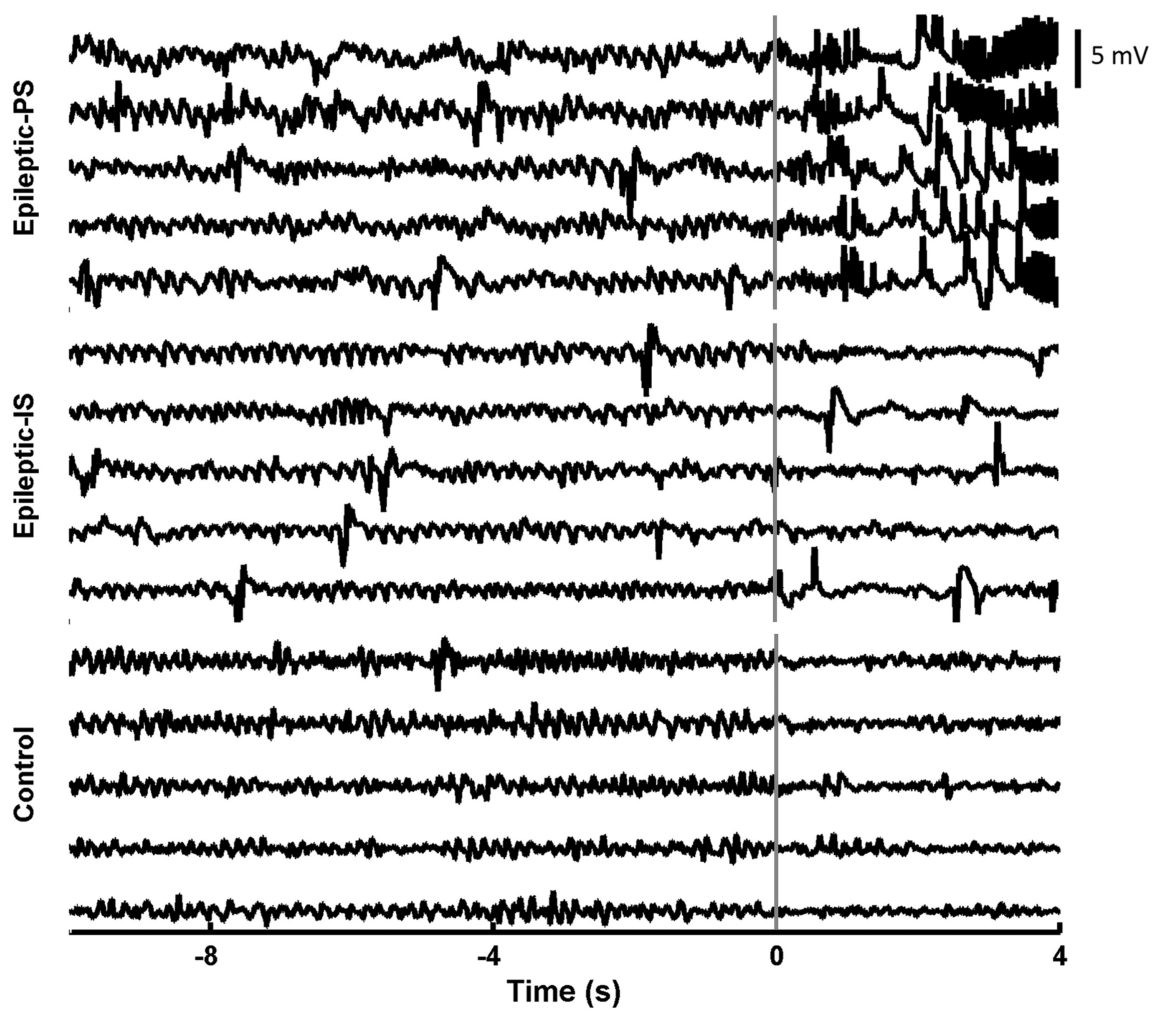

Figure 6. Characteristics of REM in control and epileptic animals. Typical transitions out of REM are shown for preseizure (PS; top) and interseizure (IS; middle) REM bouts in an epileptic animal and for a control animal (bottom). Preseizure REM bouts end in seizure and interseizure, and control REM bouts end in either NREM or Wake. Signals reflect LFPs recorded from the left dorsal hippocampus, bandpass filtered between 0.5 and $35 \mathrm{~Hz}$ and aligned to the end of the REM bout marked by the vertical line. Interictal epileptic discharges can be seen in both preseizure and interseizure REM bouts but not in control animals.

We used survival analysis to distinguish preseizure from interseizure and control REM bouts. Specifically, we calculated the hazard rate $(\lambda)$ as the instantaneous rate of transitioning out of a REM bout at time $t$, given that the REM bout has lasted up to time $t$. We required at least five transitions for each bout duration in the rate calculations, which resulted in truncation of estimates at 150 s. In Figure 7, we show the mean hazard rate and bootstrap $(n=100)$-estimated SEM rates for transitioning out of REM into Wake or NREM for the control group, $\lambda_{C-\mathrm{w} / \mathrm{n}}$ (green), into Wake or NREM for the epileptic group, $\lambda_{\mathrm{E}-\mathrm{w} / \mathrm{n}}$ (blue), and into seizure for the epileptic group, $\lambda_{\mathrm{E}-\mathrm{sz}}$ (red). The early peak in $\lambda_{\mathrm{C}-\mathrm{w} / \mathrm{n}}$ represents short REM bouts that were less frequently observed in epileptic animals. The difference in the mean amplitude of $\lambda_{\mathrm{E}-\mathrm{sz}}$ and $\lambda_{\mathrm{E}-\mathrm{w} / \mathrm{n}}$ can be attributed to the $4: 1$ ratio of interseizure-topreseizure REM bouts. More importantly, both $\lambda_{\mathrm{C}-\mathrm{w} / \mathrm{n}}$ and $\lambda_{\mathrm{E}-\mathrm{w} / \mathrm{n}}$ increase as a function of time in REM past $\sim 100 \mathrm{~s}$, whereas $\lambda_{\mathrm{E}-\mathrm{sz}}$ peaks near $80 \mathrm{~s}$ and decays for longer durations. We are most interested in significant differences in the shape of $\lambda_{\mathrm{E}-\mathrm{w} / \mathrm{n}}$ and $\lambda_{\mathrm{E}-\mathrm{sz}}$ as this would indicate unique features of the preseizure REM bout duration distribution, which may help to predict which REM bouts lead to seizure.

To assess the significance of the differences between $\lambda_{\mathrm{E}-\mathrm{sz}}$ and $\lambda_{\mathrm{E}-\mathrm{w} / \mathrm{n}}$ shapes, bootstrap techniques were used to compare grouped hazard rates with surrogate population hazard rates. Surrogate preseizure and interseizure populations with a 1:4 ratio were created by drawing from all REM bouts. The null hypothesis underlying this surrogate grouping and comparison is that all epileptic REM bouts have the same hazard rate independent of transition type and that apparent differences can be attributed to the ratio of group sizes and/or small sample sizes. Mean and SEM of these surrogate populations' hazard rates are shown in black lines in Figure 7. The point-wise significance at 10 s intervals was assessed by comparing each bootstrap hazard rate distribution with its population surrogate using a twosample Kolmogorov-Smirnov (KS) test. Asterisks in Figure 7 indicate point-wise significance at the $\alpha=0.05$ level. The hazard rate for transitioning from REM to seizure, $\lambda_{\mathrm{E}-\mathrm{sz}}$, deviates significantly from its population surrogate, particularly near $80 \mathrm{~s}$ and after $100 \mathrm{~s}$. In contrast, $\lambda_{\mathrm{E}-\mathrm{w} / \mathrm{n}}$ is close to its population surrogate estimate.

Together, these observations reject the null hypothesis that preseizure and interseizure REM bout hazard rates have the same shape. Critically, they indicate that whereas the rate to transition from REM to nonseizure states continues to increase with bout duration, the seizure transition rate peaks near $80-100 \mathrm{~s}$ and begins to decrease afterward. This implies that the underlying mechanism inducing the seizure from REM is different from that inducing the transition out of REM into a normal SOV.

\section{Discussion}

We used continuous long-term recordings and derived high-temporal-resolution SOV classifications for $19 \mathrm{~d}$ of recording and 486 seizures across six epileptic rats with the TeTX model of chronic TLE. We found that although epileptic rats spend just $6.1 \%$ of total time in REM sleep, $47 \%$ of seizures occurred during this state. In contrast, we observed that despite $39 \%$ of total time in spent in NREM sleep, only $6.8 \%$ of seizures initiated in this state. In addition, $34 \%$ of all seizure occurred during wake periods with high hippocampal theta activity $\left(\mathrm{Wake}_{\theta}\right)$. These findings support a growing body of evidence that seizures can arise from naturally occurring theta rhythms and REM in a variety of epilepsies.

Our demonstration of significantly different SOV-dependent seizure susceptibilities implies potential utility of SOV in seizure prediction algorithms. The recent publication by Cook et al. (2013) demonstrated seizure prediction capability using longterm invasive recordings from human subjects. The algorithms used in this work relied purely on classification of features derived from subdurally recorded neural activity. The seizure classifications were independent of behavioral state or time of day and were presented in terms of warnings to the subjects: low, medium, or high likelihood of seizure occurrence. A relationship between SOV and seizure frequency has been observed in many human epilepsies. Therefore, our results support the notion (Mormann et al., 2007) that incorporating SOV would yield further improvements for seizure prediction and for feedback-based seizure control schemes. This hypothesis, although not directly addressed here, can be validated for any data set that includes measurements sufficient for the scoring of both seizures and SOV with adequate temporal resolution.

We find in this model that REM is the SOV with the highest seizure susceptibility. The instantaneous seizure rate from REM is $\sim 10$ times the baseline seizure rate, and nearly half of all sei- 


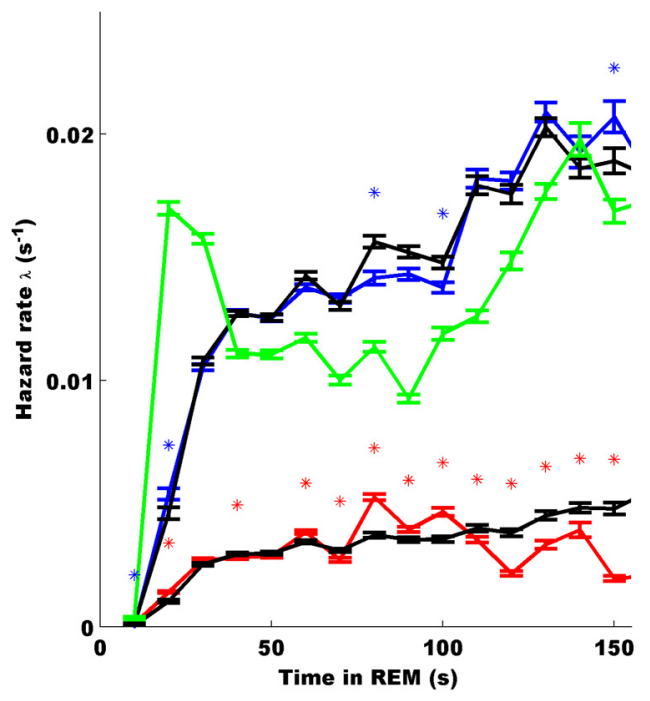

Figure 7. Hazard functions for transitioning out of REM for control and epileptic (preseizure and interseizure) REM bouts. Hazard rates for epileptic rats were calculated using a competing risk framework. Bootstrap samples drawn from the control, preseizure, and interseizure groups were used to estimate hazard rates for transitioning out of REM into Wake or NREM for the control group, $\lambda_{C-w / n}$ (green); into Wake or NREM for the epileptic group, $\lambda_{\mathrm{E}-\mathrm{w} / \mathrm{n}}$ (blue); and into seizure for the epileptic group, $\lambda_{\mathrm{E}-s \mathrm{z}}($ red). Error bars indicate SEM. To assess significance of differences in the shape of the preseizure and interseizure hazard rates, bootstrap techniques were used to compare grouped hazard rates with surrogate population hazard rates (black). Point-wise significance was assessed for each 10 s interval by comparing $\lambda_{\mathrm{E}-\mathrm{w} / \mathrm{n}}$ with its population surrogate (bottom black line) and $\lambda_{E-w / n}$ with its population surrogate (top black line) using a two-sample KS test. Asterisks indicate point-wise significance at the $\alpha=0.05$ level. The peak in $\lambda_{C-w / n}$ represents short REM bouts that were less frequent in epileptic animals. Both $\lambda_{C-w / n}$ and $\lambda_{E-w / n}$ increase as a function of time in REM past $\sim 100 \mathrm{~s}$, whereas $\lambda_{E-s z}$ is humped with a peak near $80 \mathrm{~s}$ and decays thereafter. The peak as well as the decay after $100 \mathrm{~s}$ represents significant deviations of $\lambda_{E-s z}$ from the surrogate population hazard rates.

zures emerge during REM. In human epilepsies, seizures practically never emerge from the REM phase of sleep ( $\mathrm{Ng}$ and Pavlova, 2013). But it should be noted that hippocampal activity during human REM is significantly different from that of rodent REM (Cantero et al., 2003), which is characterized by sustained rhythmic hippocampal theta oscillations. Coupled with our observation that another one-third of all seizures emerged from exploratory wake $\left(\mathrm{Wake}_{\theta}\right)$, which is also characterized by high hippocampal theta activity, our findings implicate theta rhythm as the common factor in seizure susceptibility in this model.

There are now a growing number of reports of hippocampal theta before seizure in animal models. These include the observation of hippocampal theta rhythms before spontaneous seizures in rabbits in the latter stages of a kindling model (Kitchigina and Butuzova, 2009) during head-fixed recordings, as well as prolonged theta oscillations from microelectrode recordings in pilocarpine-treated rats (Grasse et al., 2013). Our findings extend these observations with a detailed demonstration that links the preseizure theta rhythm to normal SOV-related behavior. In the case of preseizure REM, the dynamics of the sleep cycle produce the sustained theta oscillations, during which seizures emerge. The peak seizure rate from REM occurs $80-100 \mathrm{~s}$ after the onset of REM, according to our survival analysis (Fig. 7). In the case of Wake ${ }_{\theta}$, seizures were observed to emerge in the midst of exploratory wake behavior, as measured from head motion and confirmed from video. To our knowledge, there are no detailed studies on the prevalence of hippocampal theta rhythms before seizures from humans.

Our findings are in contrast to the growing consensus (Malow, 2007; Kothare and Zarowki, 2011; Derry and Duncan, 2013; Dew- olfe et al., 2013, Ng and Pavlova, 2013) that NREM and relaxed wake states permit and REM and active wake states prevent epileptic seizures. These notions emerge, in part, from seminal studies in the cat penicillin and amygdala kindling models of epilepsy by Shouse (1987) and Shouse et al. $(1989,1990,2000)$ in which a majority of seizures emerged from NREM and few seizures emerged from REM, attributed to differences in cortical synchrony between these states (Shouse et al., 1989, 2000; but see Sinha, 2011). In contrast, the interpretation that hippocampal theta is antiepileptic derives from studies of the effect of induced medial-septum theta rhythm on ongoing status epilepticus (Miller et al., 1994), modulation of electrical stimulus induced afterdischarge threshold (Kumar and Raju, 2001) or modulation of interictal epileptiform discharge frequency (Colom et al., 2006; Kitchigina and Butuzova, 2009). However, the relevance of such studies to spontaneous seizure susceptibility is indirect.

There is also the suggestion that a type of theta activity emerges before seizures as an "attempt to stop the attack" (Kitchigina and Butuzova, 2009). The theta oscillations we observe before seizures initiate with behavioral states tens of seconds before seizure onset. These preseizure theta oscillations are not differentiable from interseizure theta oscillations associated with naturally occurring SOVs that do not transition to seizure (Fig. 6). In addition, in this model we observe $\sim 20 \%$ of seizures emerging from states with no native behavioral theta rhythm. In these cases, we observe no increase in hippocampal theta just before seizure onset. Based on these observations, we hypothesize that the preseizure hippocampal theta oscillations in the TeTX model are not induced through a mechanism that attempts to stop the seizures.

Our observations that, in the TeTX model of TLE, seizure rate variations with SOV are far more dramatic than diurnal, or presumed circadian-driven (Talathi et al., 2009), variations are in contrast with the seminal publications by Quigg et al. (1998, 2000), who attributed most of the observed diurnal variation in seizure rate to diurnal/circadian oscillations. The discrepancy in our results may be attributed in part to differences between the TeTX model and the poststatus model, and its underlying mechanistic seizure susceptibilities, used by Quigg et al. (1998, 2000).

The reproducibility of the SOV-dependent seizure susceptibility across our animals, as well as similar diurnal SOV and theta dynamics between epileptic and control rats, suggests that the functioning of the SOV and theta regulatory structures in the TeTX model are substantially intact. However, the reduced time spent in REM, as well as the loss of brief $(<20 \mathrm{~s})$ REM bouts in epileptic rats, suggests some alterations in the larger hippocampal-septal-brainstem network and is consistent with observations of altered sleep dynamics in human epilepsies.

Our observations of high-seizure susceptibility during hippocampal theta oscillations, and especially during the sustained theta in REM, could be explained through a number of putative mechanisms linked to the details of the TeTX model. A full articulation of seizure mechanism will need to also explain the variable nature of seizure occurrence (why seizures only sometimes occur) as well as why the peak seizure rate is seen $80-100 \mathrm{~s}$ into a REM bout.

The hippocampal TeTX is a nonlesional model that does not produce significant or widespread cell loss (Jefferys et al., 1995; Benke and Swann, 2004; Sharma et al., 2007). The seizure focus is determined by the site of toxin injection. The circuitry involved in the generation and maintenance of hippocampal theta rhythm is complex (Pignatelli et al., 2012) with input and output path- 
ways that include the medial septum, hippocampal dentate gyrus (DG) and CA3, and entorhinal cortex.

One possible interpretation of our observations is consistent with the concept of compromised DG functioning (Lothman et al., 1992). Reduced inhibition invoked by TeTX has been shown to alter the function of the DG (Finnerty et al., 2001) feedforward-feedback granule cell and inhibitory interneuron networks (Sundströmand Mellanby, 1990). The DG filters input from the perforant path, which has maximal synaptic excitability during theta oscillations (Schall and Dickson, 2010), thereby potentially leading to increased sensitivity to theta-band input from the medial septum.

Our observations of interictal discharges during both preseizure and interseizure REM bouts (Fig. 6) support the notion of generally increased hippocampal excitability during theta rhythm. However, given the role of inhibitory interneurons in maintaining theta rhythm (Buzsaki, 2001), we were surprised by the lack of overt changes in theta oscillations of epileptic rats compared with controls.

A potential cellular-level mechanism can be derived directly from the local weakening of the inhibitory network from the toxin. To maintain balance within feedback loops during sustained theta oscillations, interneurons may fire more often to compensate for reduced transmitter output. This, in turn, would lead to a buildup of extracellular potassium, potassium depolarization block of the interneurons, and runaway excitation as observed at the cellular level in in vitro studies of Žiburkus et al. $(2006,2013)$. The time course for such a buildup would be many tens of seconds, consistent with the peak transition to seizure from REM at $80-100 \mathrm{~s}$.

This report of a novel relationship between SOV and seizure onset in the TeTX model serves as an important contribution to the growing understanding of the relationship between sleep and seizure networks. In particular, it offers a unique experimental opportunity for assessing the role of theta oscillations in seizure generation.

\section{References}

Ayala-Guerrero F, Alfaro A, Martínez C, Campos-Sepúlveda E, Vargas L, Mexicano G (2002) Effect of kainic acid-induced seizures on sleep patterns. Proc West Pharmacol Soc 45:178-180. Medline

Bazil CW, Walczak TS (1997) Effects of sleep and sleep stage on epileptic and nonepileptic seizures. Epilepsia 38:56-62. CrossRef Medline

Benke TA, Swann J (2004) The tetanus toxin model of chronic epilepsy. Adv Exp Med Biol 548:226-238. CrossRef Medline

Bokil H, Andrews P, Kulkarni JE, Mehta S, Mitra PP (2010) Chronux: a platform for analyzing neural signals. J Neurosci Methods 192:146-151. CrossRef Medline

Britton P, Whitton PS, Fowler LJ, Bowery NG (1996) Tetanus toxininduced effects on extracellular amino acid levels in rat hippocampus: an in vivo microdialysis study. J Neurochem 67:324-329. CrossRef Medline

Buckmaster PS (2004) Laboratory animal models of temporal lobe epilepsy. Comp Med 54:473-485. Medline

Buzsaki G (2001) Hippocampal GABAergic interneurons: a physiological perspective. Neurochem Res 26:889-905. Medline

Cantero JL, Atienza M, Stickgold R, Kahana MJ, Madsen JR, Kocsis B (2003) Sleep-dependent theta oscillations in the human hippocampus and neocortex. J Neurosci 23:10897-10903. Medline

Cepeda C, Tanaka T, Riche D, Naguet R (1982) Limbic status epilepticus: behavior and sleep alterations after intra-amygdala kainic acid microinjections in Papio baboons. Electroencephalogr Clin Neurophysiol 54: 603-613. CrossRef Medline

Colom LV, García-Hernández A, Castañeda MT, Perez-Cordova MG, Garrido-Sanabria ER (2006) Septo-hippocampal networks in chronically epileptic rats: potential antiepileptic effects of theta rhythm generation. J Neurophysiol 95:3645-3653. CrossRef Medline

Cook MJ, O’Brien TJ, Berkovic SF, Murphy M, Morokoff A, Fabinyi G,
D'SouzaW, Yerra R, Archer J, Litewka L, Hosking S, Lightfoot P, Ruedebusch V, Sheffield WD, Snyder D, Leyde K, Himes D (2013) Prediction of seizure likelihood with a long-term, implanted seizure advisory system in patients with drug-resistant epilepsy: a first-in-man study. Lancet Neurol 12:563-571. CrossRef Medline

Crespel A, Coubes P, Baldy-Moulinier M (2000) Sleep influences on seizures and epilepsy effects on sleep in partial frontal and temporal lobe epilepsies. Clin Neurophysiol 11 [Suppl 2]:S54-S59.

Derry CP, Duncan S (2013) Sleep and epilepsy. Epilepsy Behav 26:394-404. CrossRef Medline

Dewolfe JL, Malow B, Huguenard J, Stickgold R, Bourgeois B, Holmes GL (2013) Sleep and epilepsy: a summary of the 2011 Merrit-Puttnam symposium. Epilepsy Curr 13:42-49. CrossRef Medline

Dinner DS (2002) Effect of sleep on epilepsy. J Clin Neurophysiol 19:504513. CrossRef Medline

Empson RM, Amitai Y, Jefferys JG, Gutnick MJ (1993) Injection of tetanus toxin into the neocortex elicits persistent epileptiform activity but only transient impairment of GABA release. Neuroscience 57:235-239. CrossRef Medline

Finnerty GT, Whittington MA, Jefferys JG (2001) Altered dentate filtering during the transition to seizure in the rat tetanus toxin model of epilepsy. J Neurophysiol 86:2748-2753. Medline

Furstova J, Valenta Z (2011) Statistical analysis of competing risks: overall survival in a group of chronic myeloid leukemia patients. Eur J Biomed Inform 7:en2-en8.

Grasse DW, Karunakaran S, Moxon KA (2013) Neuronal synchrony and the transition to spontaneous seizures. Exp Neurol 248:72-84. CrossRef Medline

Herman ST, Walczak TS, Bazil CW (2001) Distribution of partial seizures during the sleep-wake cycle: differences by seizure onset site. Neurology 56:1453-1459. CrossRef Medline

Jefferys JG, Walker MC (2006) Tetanus toxin model of focal epilepsy. In: Models of seizures and epilepsy (Pitkanen A, Schwartzkroin PA, Moshe SL, eds), pp 407-414. Burlington, MA: Elsevier.

Jefferys JG, Borck C, Mellanby J (1995) Chronic focal epilepsy induced by intracerebral tetanus toxin. Ital J Neurol Sci 16:27-32. CrossRef Medline

Kitchigina VF, Butuzova MV (2009) Theta activity of septal neurons during different epileptic phases: the same frequency but different significance. Exp Neurol 216:449-458. CrossRef Medline

Kothare SV, Zarowski M (2011) Sleep and epilepsy: common bedfellows. J Clin Neurophysiol 28:101-102. CrossRef Medline

Kumar P, Raju TR (2001) Seizure susceptibility decreases with enhancement of rapid-eye-movement sleep. Brain Res 922:299-304. CrossRef Medline

Loddenkemper T, Lockley SW, Kaleyias J, Kothare SV (2011) Chronobiology of epilepsy: diagnostic and therapeutic implications of chronoepileptology. J Clin Neurophysiol 28:146-153. CrossRef Medline

Lothman EW, Stringer JL, Bertram EH (1992) The dentate gyrus as a control point for seizures in the hippocampus and beyond. Epilepsy Res Suppl 7:301-313. Medline

Mainardi M, Pietrasanta M, Vannini E, Rossetto O, Caleo M (2012) Tetanus neurotoxin-induced epilepsy in mouse visual cortex. Epilepsia 53:e132e136. CrossRef Medline

Malow BA (2007) The interaction between sleep and epilepsy. Epilepsia 48 [Suppl 9]:36-38. CrossRef Medline

Matos G, Tsai R, Baldo MV, de Castro I, Sameshima K, Valle AC (2010) The sleep-wake cycle in adult rats following pilocarpine-induced temporal lobe epilepsy. Epilepsy Behav 17:324-331. CrossRef Medline

Matos G, Tufik S, Scorza FA, Cavalheiro EA, Andersen ML (2011) Sleep, epilepsy and translational research: what can we learn from the laboratory bench? Prog Neurobiol 95:396-405. CrossRef Medline

Miller JW, Turner GM, Gray BC (1994) Anticonvulsant effects of the experimental induction of hippocampal theta activity. Epilepsy Res 18:195204. CrossRef Medline

Minecan D, Natarajan A, Marzec M, Malow B (2002) Relationship of epileptic seizures on sleep stage and sleep depth. Sleep 25:899-904. Medline

Mormann F, Andrzejak RG, Elger CE, Lehnertz K (2007) Seizure prediction: the long and winding road. Brain 130:314-333. CrossRef Medline

Ng M, Pavlova M (2013) Why are seizures rare in rapid eye movement sleep? Review of the frequency of seizures in different sleep stages. Epilepsy Res Treat 2013:932790. CrossRef Medline 
Paxinos G, Watson C (2006) The rat brain in stereotaxic coordinates, Ed 6. Burlington, MA: Academic.

Pignatelli M, Beyeler A, Leinekugel X (2012) Neural circuits underlying the generation of theta oscillation. J Physiol Paris 106:81-92. CrossRef Medline

Quigg M, Straume M, Menaker M, Bertram EH 3rd (1998) Temporal distribution of partial seizures: comparison of an animal model with human partial epilepsy. Ann Neurol 43:748-755. CrossRef Medline

Quigg M, Clayburn H, Straume M, Menaker M, Bertram EH 3rd (2000) Effects of circadian regulation and rest-activity state on spontaneous seizures in a rat model of limbic epilepsy. Epilepsia 41:502-509. CrossRef Medline

Racine RJ (1972) Modification of seizure activity by electrical stimulation. II. Motor seizure. Electroencephal Clin Neurophysiol 32:281-294. CrossRef 4110397

Schall KP, Dickson CT (2010) Changes in hippocampal excitatory synaptic transmission during colinergically induced theta and slow oscillation states. Hippocampus 20:279-292. CrossRef Medline

Schiavo G, Benfenati F, Poulain B, Rossetto O, Polverino de Laureto P, DasGupta BR, Montecucco C (1992) Tetanus and botulinum-B neurotoxins block neurotransmitter release by proteolytic cleavage of synaptobrevin. Nature 359:832-835. CrossRef Medline

Sharma AK, Reams RY, Jordan WH, Miller MA, Thacker HL, Snyder PW (2007) Mesial temporal lobe epilepsy: pathologies, induced rodent models and lesions. Toxicol Pathol 35:984-999. CrossRef Medline

Shouse MN (1987) Thalamacortical mechanisms of state-dependent seizures during amygdala kindling and systematic penicillin epilepsy in cats. Brain Res 425:198-203. CrossRef Medline

Shouse MN, Siegel JM, Wu MF, Szmusiak R, Morrison AR (1989) Mechanisms of seizure suppression during rapid-eye-movement (REM) sleep in cats. Brain Res 505:271-282. Medline
Shouse MN, Langer JV, Dittes PR (1990) Spontaneous sleep epilepsy in amygdala-kindled kittens: a preliminary report. Brain Res 535:163-168. CrossRef Medline

Shouse MN, Farber PR, Staba RJ (2000) Physiological basis: how NREM sleep components can promote and REM sleep components can suppress seizure discharge propagation. Clin Neurophysiol 111:S9-S18. CrossRef Medline

Sinha SR (2011) Basic mechanisms of sleep and epilepsy. J Clin Neurophysiol 28:103-110. CrossRef Medline

Soltesz I, Staley K (2008) Computational neuroscience in epilepsy. Burlington, MA: Academic.

Sunderam S, Chernyy N, Peixoto N, Mason JP, Weinstein SL, Schiff SJ, Gluckman BJ (2007) Improved sleep-wake and behavior discrimination using MEMS accelerometers. J Neurosci Methods 163:373-383. CrossRef Medline

SundströmLE, Mellanby JH (1990) Tetanus toxin blocks inhibition of granule cells in the dentate gyrus of the urethane-anesthetized rat. Neuroscience 38:621-627. CrossRef Medline

Talathi SS, Hwang DU, Ditto WL, Mareci T, Sepulveda H, Spano M, Carney PR (2009) Circadian control of neural excitability in an animal model of temporal lobe epilepsy. Neurosci Lett 455:145-149. CrossRef Medline

Vreugdenhil M, Hack SP, Draguhn A, Jefferys JG (2002) Tetanus toxin induces long-term changes in excitation and inhibition in the rat hippocampal CA1 area. Neuroscience 114:983-994. CrossRef Medline

Žiburkus J, Cressman JR, Barreto E, Schiff SJ (2006) Interneuron and pyramidal cell interplay during in vitro seizure-like events. J Neurophysiol 95:3948-3954. CrossRef Medline

Žiburkus J, Cressman JR, Schiff SJ (2013) Seizures as imbalanced up states: excitatory and inhibitory conductances during seizure-like events. J Neurophysiol 109:1296-1306. CrossRef Medline 